\title{
Relationship between multiple type II solar radio bursts and CME observed by STEREO/SECCHI
}

\author{
K.-S. Cho ${ }^{1}$, S.-C. Bong ${ }^{1}$, Y.-J. Moon ${ }^{2,5}$, A. Shanmugaraju ${ }^{3}$, R.-Y. Kwon ${ }^{4,5}$, and Y. D. Park ${ }^{1}$ \\ 1 Korea Astronomy and Space Science Institute, Hwaamdong, Yuseong-Gu, 305-348 Daejeon, Republic of Korea \\ e-mail: kscho@kasi.re.kr \\ 2 Dept. of Astronomy \& Space Science, Kyung Hee University, Yongin-shi, 446-701 Kyungki-do, Republic of Korea \\ 3 Department of Physics, Arul Anandar College, 625-514 Karumathur, Madurai, India \\ 4 Astronomy Program, Department of Physics and Astronomy, Seoul National University, Republic of Korea \\ 5 NASA Goddard Space Flight Center, Greenbelt, MD 20771, USA
}

Received 13 August 2010 / Accepted 9 February 2011

\section{ABSTRACT}

\begin{abstract}
Aims. Two or more type II bursts are occasionally observed in close time sequence during solar eruptions, which are known as multiple type II bursts. The origin of the successive burst has been interpreted in terms of coronal mass ejections (CMEs) and/or flares. Detailed investigations of the relationship between CMEs and the bursts enable us to understand the nature of the multiple type II bursts. In this study, we examine multiple type II bursts and compare their kinematics with those of a CME occurring near the time of the bursts. Methods. To do this, we selected multiple type II bursts observed by the Culgoora radiospectrographs and a limb CME detected in the low corona field of view $\left(1.4-4 R_{\mathrm{S}}\right)$ of a STEREO/SECCHI instrument on December 31, 2007. To determine the 3D kinematics of the CME, we applied the stereoscopic technique to the STEREO/SECCHI data.

Results. Our main results are as follows: (1) the multiple type II bursts occurred successively at ten minute intervals and displayed various emission structures and frequency drifting rates; (2) near the time of the bursts, the CME was observed by STEREO and SOHO simultaneously, but no evidence of other CMEs was detected; (3) inspection of the 3D kinematics of the CME using the stereoscopic observation by STEREO/SECCHI revealed that the CME propagated along the eastward radial direction as viewed from the Earth; (4) very close time and height associations were found between the CME nose and the first type II burst, and between CME-streamer interaction and the second type II burst.

Conclusions. On the basis of these results, we suggest that a single shock in the leading edge of the CME could be the source of the multiple type II bursts and support the notion that the CME nose and the CME-streamer interaction are the two main mechanisms able to generate the bursts.
\end{abstract}

Key words. shock waves - Sun: coronal mass ejections (CMEs) - Sun: radio radiation - Sun: corona

\section{Introduction}

A metric type II solar radio burst is a slow frequency-drifting form of radio emission, and has been interpreted as a signature of the magnetohydrodynamic (MHD) shock propagating outward through the solar corona $\left(1-5 R_{\mathrm{S}}\right)$. Since the first discovery of the burst by Payne-Scott et al. (1947), there has been debate about the origin of the type II burst, in particular whether it has a flare or CME origin (Wagner \& McQueen 1983; Gosling 1993; Gosling \& Hundhausen 1995; Svestka 1995; Dryer 1996; Gopalswamy et al. 1998; Cliver et al. 1999; Mancuso \& Raymond 2004; Cliver et al. 2005).

Robinson \& Sheriden (1982) reported that occasionally two or more type II bursts are observed in close time-sequence during solar eruptions (about $20 \%$ of all the type IIs). The multiple type II bursts consist of multiple bands with different frequency drift rates and frequency bands, which are different from the normal fundamental-harmonic or band-split relationship (Nelson $\&$ Melrose 1985). Along with the aforementioned controversy, the successive type II bursts has been interpreted from different points of view. One view is that they are caused by successive bursts ignited by either flares (e.g. Gergely et al. 1984) or CMEs (e.g. Raymond et al. 2000). This view maintains that multiple type II bursts are caused by a single shock travelling through different coronal structure since there are no multiple disturbances.
Another view (Reiner et al. 2001; Shanmugaraju et al. 2005) is that two coronal shocks are generated from different sources; one burst is due to flares and the other caused by CME. In particular, Shanmugaraju et al. (2005) suggested that flares and CMEs could both be sources of multiple type II bursts by reporting that there is no event associated with multiple flares or multiple CMEs, and no correlation between two shock speeds.

Owing to a lack of spatial overlap between type II burst and CME observation, their direct comparison has rarely been reported. Cho et al. (2007, 2008) investigated the type IIassociated CMEs that were observed with a ground-based MK4 coronameter. They concluded based on a coronal density measurement that the type II bursts might be generated at the interface of the CME flank and the streamer. Ramesh et al. (2010) inspected the seven near-limb type II burst obtained by the Gauribidanur radioheliograph (GRH, Ramesh et al. 1998) between 1997 and 2005 and reported that the type II associated CMEs are largely accelerated before the type II bursts start. They noted that the present spatial resolution of the GRH is insufficient to precisely locate the type II bursts in low corona. Since SOHO/LASCO (Large Angle Spectrographic Coronagraph) covers high coronal observations above $3 R_{\mathrm{s}}$ (Brueckner et al. 1995), the inner coronagraph (COR1) of the Sun Earth Connection Coronal and Heliospheric Investigation 
(SECCHI, Howard et al. 2008) instrument suite on board the Solar TErrestrial RElations Observatory (STEREO, Kaiser et al. 2008) is the only space coronagraph that allows us to investigate the relationship between CME and metric type II burst with a small coronal field of view (1.4-4 $R_{\mathrm{S}}$ ), high time cadence (5 or $10 \mathrm{~min}$ ), and spatial resolution (3'.75). Comparison between the COR1 CME heights and type IIs was first done by Gopalswamy et al. (2009).

We studied the multiple type II bursts observed with the Culgoora radio spectrograph on December 31, 2007. A first identification of the multiple bursts was made by one of e-CALLISTO network installed in the Republic of Korea (Bong et al. 2009) as an activity of IHY2007 for 24-h monitoring of solar radio emissions throughout the world (Benz et al. 2009). One advantage of our dataset is that the multiple type II-associated CME was observed by the SECCHI/COR1, which makes it possible to determine the $3 \mathrm{D}$ kinematics of the CME in the low corona and inspect the relationship between the CME and the multiple type II bursts in detail. In this study, we compared the type II burst location and kinematics with those of CMEs observed by the SECCHI/COR1. For this, we estimated the type II burst height from drifting emission frequency by using a coronal density model. The CME centroid, CME-streamer interaction height, and their propagation direction was deduced by applying the 3D reconstruction method (Kwon et al. 2010) to the SECCHI/COR1 data.

The paper is organized as follows. In Sect. 2, we describe the multiple type II bursts observed by e-CALLISTO and Culgoora radio spectrographs, and the three-dimensional kinematics of CME deduced from the STEREO/SECCHI-COR1 in detail. We present a kinematic comparison of the multiple type II bursts and the CME to interpret the source of the successive type II bursts in Sect. 3. A brief summary is delivered in Sect. 4.

\section{Data and analysis}

\subsection{Multiple type II bursts}

Since launching e-CALLISTO observations in Daejeon in August 2007, two successive type II radio bursts, which are known as multiple type II bursts, were observed by eCALLISTO on December 31, 2007 as shown in Fig. 1. The bursts show the characteristics of multiple type II bursts reported by Robinson \& Sheriden (1982) and Nelson \& Melrose (1985), such as a close time sequence of 10 min and multiple bands with different frequency drift rates. The first type II burst (left panel) started at 00:55 (UT) from $80 \mathrm{MHz}$ with a frequency drift rate of $-0.1 \mathrm{MHz} / \mathrm{s}$, and the second burst (right panel) appeared at 01:05 (UT) from $75 \mathrm{MHz}$ with a frequency drift rate of $-0.07 \mathrm{MHz} / \mathrm{s}$. Owing to strong artificial radio interferences at the Daejeon station and limited observation frequency $(45-450 \mathrm{MHz})$, the eCALLISTO failed to derive the detailed structure of the successive type II bursts.

The detailed structure of the burst can be found in the dynamic spectrum of Culgoora solar spectrograph (Prestage et al. 1994) and STEREO/SWAVES instrument (Bougeret et al. 2008), as shown in Fig. 2. The upper panel of Fig. 2 is the dynamic spectrum of the Culgoora radiospectrograph in the frequency range $18-100 \mathrm{MHz}$, and the lower panel is the dynamic spectrum of STEREO/WAVES (SWAVES) instrument in the frequency range of $3-16 \mathrm{MHz}$. We note that the frequency axis in Fig. 1 is in the opposite direction of that of Fig. 2. The first type II burst occurring at 00:50 UT displays both fundamental and harmonic emission each with a band-split structure. White plus symbols
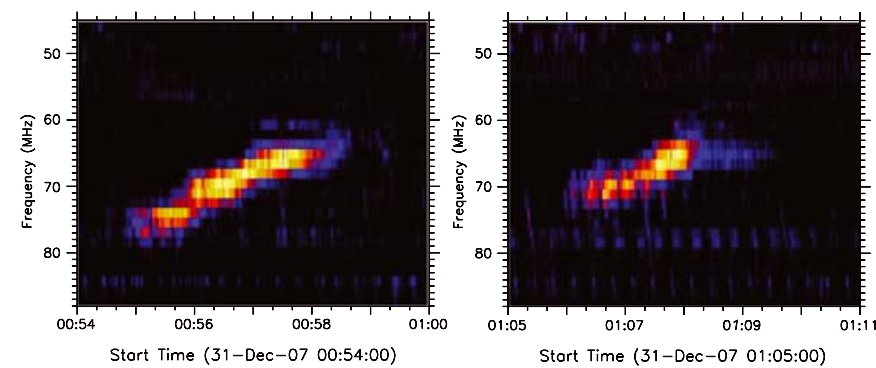

Fig. 1. e-CALLISTO dynamic spectrum of the first type II burst (left) and the second type II burst (right) of the multiple type II bursts. The second type II burst starts 10 min after the onset of the first type II burst.

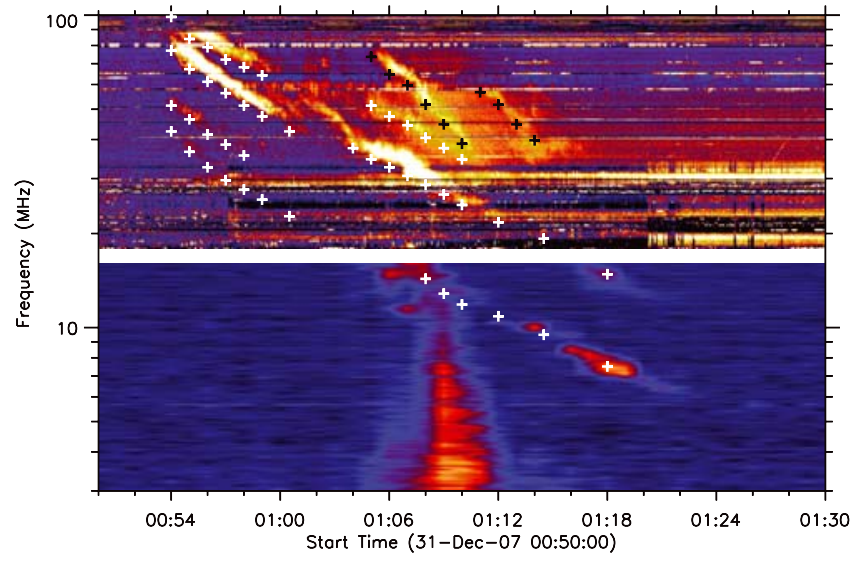

Fig. 2. Dynamic spectrum of the multiple type II bursts obtained by a Culgoora radiospectrograph (upper) and STEREO/WAVES instrument (lower). White plus symbols denote the radio emission strips of the fundamental and harmonic components of the first type II burst, and black plus symbols indicate emission strips of the fundamental and harmonic components of the second type II burst. Note that first type II has a band-splitting structure consisting of lower and upper bands.

indicate the emission trace of the first type II burst for the lower and upper bands in both emission, respectively. We note that the lower band of the fundamental emission was detected continuously by the SWAVES instrument in the decametric range, as shown in the lower panel of Fig. 2. The fundamental and harmonic emission of the second type II burst can be found in the upper spectrum of Fig. 2. As for the first type II, we can trace the fundamental and harmonic emission of the second type II. It is marked by black plus symbols. By comparing the spectra from e-CALLISTO and Culgoora spectrographs, we identified that the first and the second type II bursts appearing in the eCALLISTO spectrum (Fig. 1) correspond to the low band of the harmonic component of the first type II burst and the fundamental component of the second type II burst in the Culgoora spectra. A remarkable feature in Fig. 2 is that after starting the second type II, the SWAVES instrument detected a type III burst $(<10 \mathrm{MHz})$, which is usually known as radio emission by an electron beam that propagates along an open magnetic-field line reaching out from the Sun to the interplanetary space.

\subsection{3-D CME kinematics}

By inspecting data from SOHO and STEREO observations near the time of the multiple type II bursts, we found that the CME occurred on the east limb and there was no evidence of another CME associated with the type II bursts. The CME erupted around 00:50 UT and was followed by a C-class flare. 


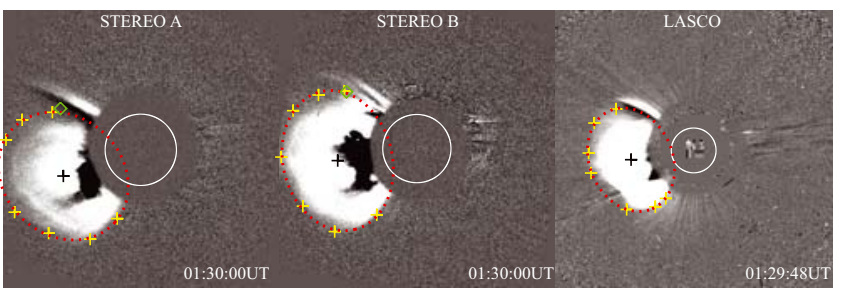

Fig. 3. CME seen from COR 1 on board STEREO A (left), STEREO $\mathrm{B}$ (middle), and LASCO C2 on board SOHO (right) at 01:30 UT. The black plus symbol denotes the centroid of the CME, which is derived from a red dot ellipse by applying the curve fitting method. The yellow plus and the green diamond symbols are the CME leading edge and CME-streamer interaction point determined by eye-detection, respectively. Here and in the following figures the circle indicates the size and position of the limb of the visible sun.

Fortunately, the CME was detected by STEREO/SECCHI and SOHO/LASCO simultaneously at 01:30 UT, which allowed us to inspect the 3D structure of the CME. Figure 3 shows the CME and a nearby streamer $\left(\mathrm{PA} \sim 60^{\circ}\right.$ ) observed by the STEREO Ahead (SA), STEREO Behind (SB), and SOHO. From all three coronagraphs in $\mathrm{SA}, \mathrm{SB}$, and $\mathrm{SOHO}$, the streamer deflection by the expanding CME can be clearly seen, which is similar to the observation by Cho et al. (2008), who used ground-based MK4 coronagraph data. They reported that the CME flank interacting with the streamer could be the place where the type II emission is generated since the high density streamer provides a good environment (low Alfvénic region) for coronal shocks. The streamer is the high density region for which the Alfven speed may be less, and the shocks can easily become super-Alfvénic in this region.

As shown in Fig. 4, the CME flank and the streamer interaction can be seen from the SECCHI/COR1 image of SB satellite taken at 01:15 UT. Red and green dotted lines and numbers denote the position angles of the CME nose and the streamer. The position angle of the streamer is deduced from the image before the CME appearance. Thus, we speculate that the first contact of the CME with the streamer occurred between 01:00 UT and 01:15 UT, which corresponds to the start times of the second type II burst and the type III burst, as shown in Fig. 2.

The separation angle between SA and SB is about $44^{\circ}$. The shape and nose heights of the CME are different depending on the viewing direction of all three coronagraphs. Figure 5 shows a comparison of CME leading edges observed by SA, SOHO, and SB satellites depending on different satellite position angles with respect to the Earth. The estimated heights from different views by $\mathrm{SA}, \mathrm{SOHO}$, and $\mathrm{SB}$ are $4.0,4.7$, and $4.1 R_{\mathrm{s}}$, respectively. This measurement shows that the projection effect was lowest for the SOHO view compared to other views from the STEREO satellites. We confirmed the above argument again by applying the ice cream cone model of CME (Xue et al. 2005) to the LASCO CME data and found that the source longitude of the CME is E89. These results are consistent with the study by Gopalswamy et al. (2009), who reported the expected source locations (E102, E81, and E58) of the CME deduced from the different views of $\mathrm{SA}, \mathrm{SOHO}$, and $\mathrm{SB}$, respectively.

One of the main objectives of this study is to determine the CME heights and compare them with the emission heights of the multiple type II bursts that can be deduced by applying a coronal electron density model (e.g. Newkirk model). The heighttime-analysis of the CME and the multiple type II bursts would be useful in deciphering the relationship between them, which has not yet been resolved (e.g. Shanmugaraju et al. 2005). In
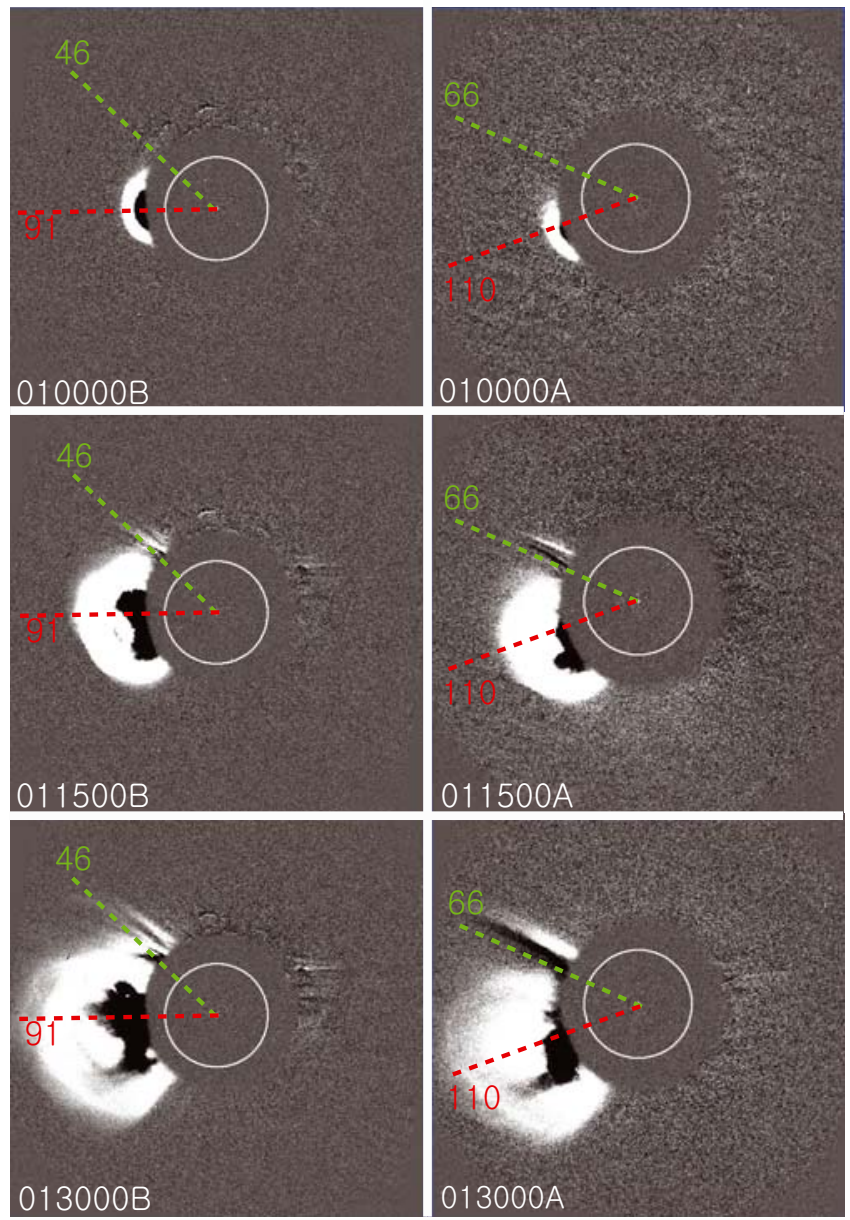

Fig. 4. Pre-event subtracted images of the expanding CME and nearby streamer between 01:00 and 01:30 UT seen from COR1 instruments onboard STEREO B (left) and STEREO A (right) satellites. Red and green dotted lines and numbers denote the position angles of the CME nose and the streamer.

principle, the location of an object can be determined by the triangulation of observations from two view points. A stereoscopic observation of the STEREO/SECCHI allows us to obtain 3D kinematics of CME as well as accurate determination of the CME-streamer interaction point. Kwon et al. (2010) developed the $3 \mathrm{D}$ reconstruction method to determine the height of EUV bright points using data taken by the STEREO/SECCHI. We adopted their method and determined the 3D kinematics of the CME by assuming that the CME had an elliptical structure. In Fig. 3, yellow pluses indicate the CME fronts determined by eye-detection, and the red dotted circle and black plus denote the deduced ellipse and its centroid by applying the MPFIT curvefitting method (Markwardt 2009).

To get a centroid of the CME in three-dimensional space, we can define two lines of sight passing through the ellipse center and observers (SA and SB). In general, the two lines skew each other with non-zero minimum distance, so that we can determine two points on these lines, minimizing the distance between them. We can then assume that their mean position is the centroid. Likewise, we can also determine the CME-streamer interaction height from two interaction points (diamonds) seen from SA and SB, as shown in the left and middle panels of Fig. 3. In the case of the CME nose, the projected leading edge at different viewing angles corresponds to different points on the nose surface so that we cannot apply the above-mentioned reconstruction 


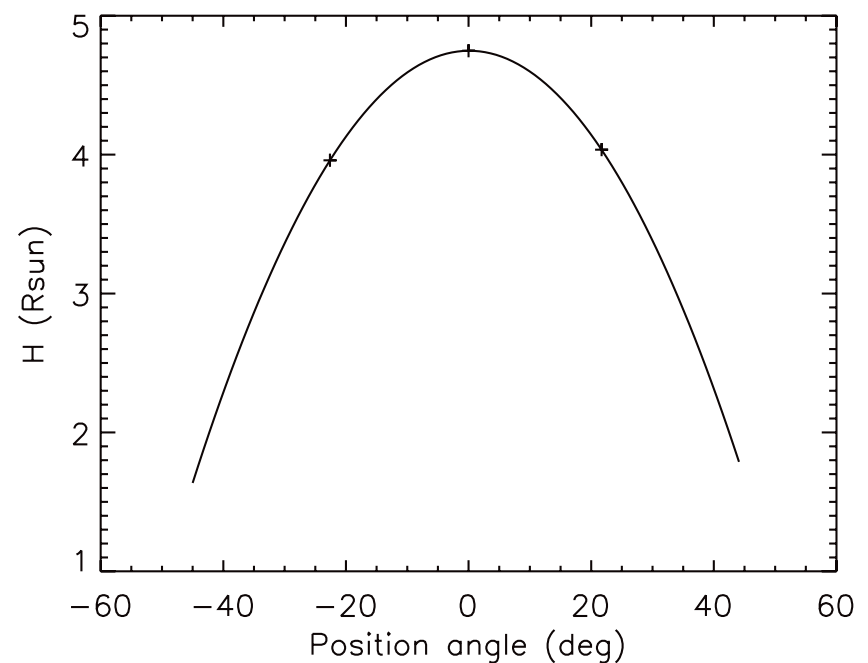

Fig. 5. Height comparison of the CME leading edge determined at 01:30 UT from STEREO A (SA), STEREO B (SB), and SOHO observations with different view of coronagraphs. SA was $21^{\circ}$ ahead of $\mathrm{SOHO}$, and $\mathrm{SB}$ was $23^{\circ}$ behind $\mathrm{SOHO}$ when they detected the CME.
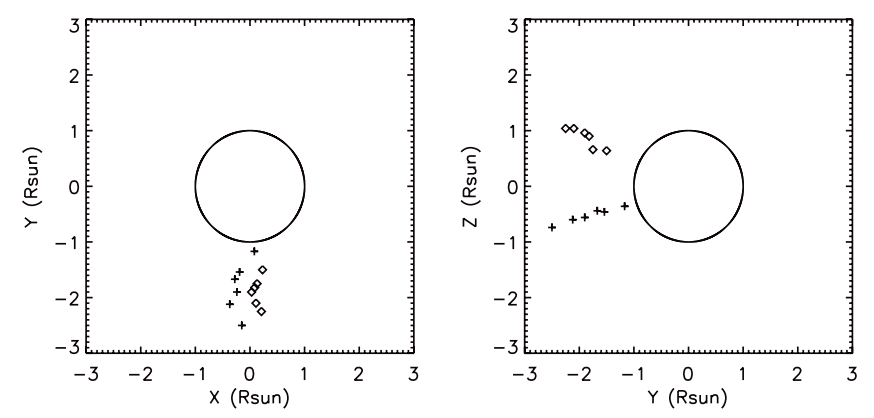

Fig. 6. Tracking of the centroid (plus symbol) of the CME and the CMEstreamer interaction position (diamond symbol) seen from the $X Y$ plane (left) and $Y Z$ plane (right) of the heliocentric coordinate. The centroid and the interaction points are determined by using the $3 \mathrm{D}$ reconstruction method.

method, which requires the same point seen from different observers. Figure 6 shows the tracking of the centroid (plus) of the $\mathrm{CME}$ and $\mathrm{CME}$-streamer interaction point (diamond). In the top view ( $X-Y$ plane) of the heliocentric coordinate, the centroid of CME is located behind the CME-streamer interaction points seen from the Earth and propagates in an eastward direction. The side view ( $Y-Z$ plane) shows that the centroid and interaction point of the CME propagate eastward but in different directions.

\section{Results and discussion}

\subsection{First type /l burst}

Our inspection of the 3D kinematics of the CME using the stereoscopic observation reveals that the CME propagates along the radial direction in the plane of the sky as viewed from the Earth. Thus, the CME observed by the SOHO/LASCO was almost devoid of projection effects, and the event is very useful for investigating the relationship between the CME and multiple type II bursts by comparing their heights.

Since the drifting stripes of metric type II emission are known as the signature of coronal shock waves, radio emission frequencies could be converted into emission heights of the shock by adopting a coronal density model. By comparing the heights of type II shocks estimated from the frequency drift of type II emission with the heights of CME fronts, we could

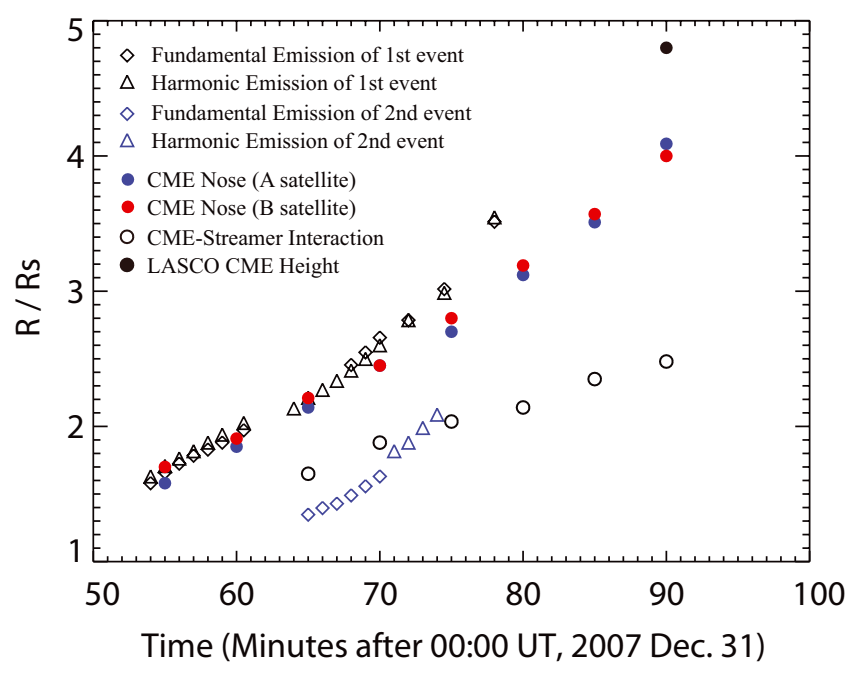

Fig. 7. Height comparison of CME with emission of type II burst. The CME data points are from STEREO COR1 and LASCO C2 cornagraphs. Circles denote the CME-streamer interaction height (open) and CME leading edges determined by using SA (filled blue), SB (filled red), and LASCO (filled black). Triangle and diamond symbols denote the emission heights of type II burst inferred from the fundamental band and harmonic band, respectively, for the first type II burst (black) and the second type II burst (blue).

simply examine whether the type II emission is generated by the shock ahead of a CME nose or not. To do so, we selected the lower bands of the fundamental and harmonic components of the first type II burst among the white plus strips in Fig. 2 and estimated the shock heights by using one-fold Newkirk coronal density model (Newkirk et al. 1961). In Fig. 7, we compares the heights for the CME nose and first type II emission. Black diamonds and triangles denote the emission heights of the type II burst estimated from the fundamental (diamond) and harmonic (triangle) components. We found that the heights for different bands are similar to each other and comparable to the heights of the CME nose below $2.5 R_{\mathrm{s}}$ measured from SA and SB. The difference between the CME nose and first type II emission becomes larger as the height increases above $2.5 R_{\mathrm{s}}$, which may be related to the projection effects of the CME seen from SA and SB. As mentioned before, we cannot obtain a reliable nose height of the CME by using the $3 \mathrm{D}$ reconstruction method so that we have to use the leading edge obtained by the images from $\mathrm{SA}$ and SB instead of applying the $3 \mathrm{D}$ reconstruction model. It is noted that there is a large difference between the CME nose heights seen by STEREO and SOHO at 10:30 UT, but the extrapolated type II emission height seems to be comparable with the height of the CME nose obtained by SOHO at 10:30 UT. Summing up, we found a very close time and height association between the CME nose and the first type II, supporting the coronal shock generation near the CME leading edge.

\subsection{Second type II burst}

As mentioned before, we found that the CME expands and sweeps the helmet streamer aside at the starting time of the second type II. While sweeping across the streamer, type III burst was detected by the SWAVES instrument. We speculate that the type III burst could be a signature of the CME-streamer interaction and that the second type II could be generated by the interaction between CMEs and helmet streamers, as suggested by Reiner et al. (2003) and reported by Vourlidas et al. (2003) and Cho et al. (2008). To investigate an association of the second 
type II with the CME, we compared the type II emission height with the CME streamer interaction height as shown in Fig. 7. We applied the Newkirk density model to the emission frequencies marked by the black plus symbols, shown in the dynamic spectrum of Fig. 2. Unlike the CME leading edge, we were able to select a point of the interaction so that we could apply the $3 \mathrm{D}$ reconstruction method and determine a CME-streamer interaction height from the SA and SB data. The heights of the above deduced points are plotted as a function of time in Fig. 7. Open circles denote the interaction height, which is comparable to the type II heights from fundamental (blue diamond) and harmonic (blue triangle) symbols. Figure 7 shows that the second type II heights are very similar to the interaction heights between the CME flank and the high density streamer, and that the emission times are nearly coincident with the interaction times. This implies that the second type II burst was generated at the CME flank. It is likely that the relatively slow CME flank could also form a shock at the location of the streamer interface because of the low local Alfvén speed at the high density streamer. This would agree with the recent theoretical investigation of the type II burst onset by Lin et al. (2006).

\section{Summary}

Since the first report of multiple type II burst observation by Robinson \& Sheriden (1982), several interpretations of the successive type IIs have been proposed. One is a single shock traveling through different coronal structures, and the other is two or more shocks from different sources. To decipher this issue, we have examined the multiple type II bursts and compared their kinematics with the CME occurring near the time of the bursts. We selected the multiple type II bursts observed on December 31, 2007 using the Culgoora radiospectrographs and an east-limb CME detected by the STEREO/SECCHI instruments. To determine the 3D kinematics of the CME in detail, we applied the stereoscopic technique developed by Kwon et al. (2010) to the STEREO/SECCHI data. Our main results can be summarized as follows:

(1) We found that the multiple type II bursts consist of two type IIs that occurred successively with ten-minute intervals with different emission structures and frequency drifting rates. In particular, the SWAVES instrument detected a type III burst with fast frequency drifting from $10 \mathrm{MHz}$ to $1 \mathrm{MHz}$ occurring just before the start time of the second type II.

(2) During multiple type II bursts, a CME was detected by STEREO/SECCHI and SOHO/LASCO simultaneously, and no evidence of another CME was found. Our inspection of the 3D kinematics of the CME using the stereoscopic observation reveals that the CME propagates along the eastward radial direction as viewed from the Earth, which is very useful for inspecting the relationship between the CME and type II radio burst by their kinematics comparing.

(3) We found very close time and height associations between the CME nose and the first type II burst and between the CME-streamer interaction and the second type II burst. There is a streamer deflection just after the CME impinges on the streamer. Interestingly, it was found that the second type II burst and type III burst start to appear when the CME flank interacts with the streamer. It is likely that electrons trapped by a flux rope of the CME are escaping along the open field formed in the helmet streamer when the CME flank contacts the streamer and generates the type III burst.
Our results show that a single shock at the leading edge of a CME could be the source of the multiple type II bursts: one burst is from the CME nose, which is the fast part of CME, and the other burst is from the CME flank interacting with high density helmet streamer, which provides the favorable condition of low Alfven speed because of the high density. This is consistent with the theoretical investigation of Lin et al. (2006) and observations of Reiner et al. (2003), Vourlidas et al. (2003), Cho et al. (2008), and Ramesh et al. (2010). However, it is not clear how general our conclusion is for multiple or non-CME nose shocks in the low corona. Further studies are needed to illuminate this problem by using more multiple type II events during solar maximum with a STEREO observation that is suited to the observation of the low corona where type II bursts are emitted.

Acknowledgements. This work was supported by the Development of Korean Space Weather Center, a project of KASI, and the KASI basic research fund. Y.J.M. was supported by the WCU program (No. R31-10016) though the National Research Foundation of Korea funded by the Ministry of Education, Science and Technology and by the Korea Research Foundation Grant funded by the south Korean Government (MOEHRD, Basic Research Promotion Fund) (KRF-2008-314-C00158, 20090071744 and 20100014501).

\section{References}

Benz, A. O., Monstein, C., Meyer, H., et al. 2009, Earth Moon Planet, 104, 277 Bong, S.-C., Kim, Y.-H., Roh, H., et al. 2009, J. Korean Astron. Soc., 42, 1 Bougeret, J. L., Goetz, K., Kaiser, M. L., et al. 2008, Space Sci. Rev., 136, 487 Brueckner, G. E., Howard, R. A., Koomen, M. J., et al. 1995, Sol. Phys., 162, 357

Cho, K.-S., Lee, J., Moon, Y.-J., et al. 2007, A\&A, 461, 1121

Cho, K.-S., Bong, S.-C., Kim, Y.-H., et al. 2008, A\&A, 491, 873

Cliver, E. W., Webb, D. F., \& Howard, R. A. 1999, Sol. Phys., 187, 89

Cliver, E. W., Nitta, N. V., Thompson, B. J., \& Zhang, J. 2005, Sol. Phys., 225, 105

Dryer, M. 1996, Sol. Phys., 169, 421

Gergely, T. E., Kundu, M. R., Wu, S. T., et al. 1984, AdSR, 4, 283

Gopalswamy, N., Kaiser, M. L., Lepping, R. P., et al. 1998, J. Geophys. Res., 103,307

Gopalswamy, N., Thompson, W. T., Davila, J. M., et al. 2009, Sol. Phys., 259, 227

Gosling, J. T. 1993, J. Geophys. Res., 98, 18937

Gosling, J. T., \& Hundhausen, A. J. 1995, Sol. Phys., 160, 57

Howard, R. A., Moses, J. D., Vourlidas, A., et al. 2008, Space Sci. Rev., 136, 67

Kaiser, M. L., Kucera, T. A., Davila, J. M., et al. 2008, Space Sci. Rev., 136, 5

Kwon, R.-Y., Chae, J., \& Zhang, ApJ. 2010, 714, 130

Lin, J., Mancuso, S., \& Vourlidas, A. 2006, ApJ, 649, L1110

Mancuso, S., \& Raymond, J. C. 2004, A\&A, 413, 363

Markwardt, C. B. 2009, in ASP Conf. Ser., ed. A. B. David, D. Daniel, \& D. Patrick, 411, 251

Nelson, G. J., \& Melrose, D. B. 1985, in Solar Radio Physics, ed. D. J. McLean, \& N. R. Labrum, Cambridge, New York, 333

Newkirk, G. Jr. 1961, ApJ, 133, 983

Payne-Scott, R., Yabsley, D. E., \& Bolton, J. G. 1947, Nature, 160, 256

Prestage, N. P., Luckhurst, R. G., Paterson, B. R., Bevins, C. S., \& Yuile, C. G. 1994, Sol. Phys., 150, 393

Ramesh, R., Subramanian, K. R., Sundararajan, M. S., \& Sastry, Ch. V. 1998, Sol. Phys., 181, 439

Ramesh, R., Kathiravan, C., Kartha, Sreeja, S., \& Gopalswamy, N. 2010, ApJ, 712,188

Raymond, J. C., Thompson, B. J., St. Cyr, O. C., et al. 2000, Geophys. Res. Lett., 27, 1439

Reiner, M. J., Kaiser, M. L., Gopalswamy, N., et al. 2001, J. Geophys. Res., 106, 25279

Reiner, M. J., Vourlidas, A., Cyr, O. C. St., et al. 2003, ApJ, 590, 533

Robinson, R. D., \& Sheridan, K. V. 1982, PASA, 4, 392

Shanmugaraju, A., Moon, Y.-J., Cho, K.-S., et al. 2005, Sol. Phys., 232, 87

Svestka, Z. 1995, Sol. Phys., 160, 53

Vourlidas, A., Wu, S. T., Wang, A. H., Subramanian, P., \& Howard, R. A. 2003, ApJ, 598, 1392

Wagner, W. J., \& MacQueen. R. M. 1983, A\&A, 120, 136

Xue, X. H., Wang, C. B., \& Dou, X. K. 2005, J. Geophys. Res., 110, A08103 